\title{
Cómo transmitir la evidencia a los pacientes: El médico como comunicador de riesgo
}

\author{
Philippa Moore ${ }^{1,2}$, Solange Rivera $M^{1,2}$, Javiera Corbalán $\mathrm{P}^{1}$. \\ Communicating evidence \\ to the patients: Physicians \\ as risk communicators
}

$\mathrm{E}$ $\mathrm{n}$ artículos previos hemos entregado nociones generales acerca de la Medicina Basada en Evidencia (MBE): sugerencias sobre cómo formular preguntas, llevar a cabo búsquedas, realizar un análisis crítico de la literatura y aplicar los resultados a nuestros pacientes ${ }^{1,2}$. Todos estos aspectos están centrados en cómo el médico puede utilizar la mejor evidencia disponible para disminuir la incertidumbre frente a una situación clínica. En este artículo nos referiremos al proceso de entrega de información, pilar fundamental de la relación médico-paciente, y a su relevancia en relación a los resultados de la práctica clínica.

La relación médico-paciente y la medicina en su totalidad, han evolucionado ${ }^{3}$. Cambios sociales de las últimas décadas, como el mayor nivel educacional y el mayor acceso a la información, entre otros, se han traducido en una pérdida de la influencia normativa que tenían los médicos. Los pacientes son más activos y demandantes en la recepción de la información ${ }^{4}$. En consecuencia, la transmisión de información clínica cobra una importancia cada vez mayor entre profesionales y usuarios de las ciencias de la salud.

\section{¿CUÁNTA INFORMACIÓN ENTREGAN LOS MÉDICOS A SUS PACIENTES?}

La comunicación clínica representa hoy uno de los aspectos fundamentales de la atención en todas las áreas de la medicina. Supone precisamente el método a través del cual dicha atención se produce y son ya muchos los estudios bien diseñados que muestran que una comunicación clínica efectiva afecta de forma positiva al proceso diagnósticoterapéutico ${ }^{5}$. Sin embargo, los médicos entregan poca información a sus pacientes y éstos quisieran saber más ${ }^{6-9}$. Análisis de entrevistas médicas han mostrado que la discusión acerca del plan de tratamiento suele ocupar en promedio no más de $5 \%$ de la entrevista ${ }^{10,11}$ (de los 20 min que estamos con el paciente sólo dedicamos 1 min para discutir el plan de manejo con él) y aproximadamente en

${ }^{1}$ Unidad de Medicina Basada en Evidencia, Pontificia Universidad Católica de Chile. ${ }^{2}$ Departamento de Medicina Familiar, Pontificia Universidad Católica de Chile. Santiago de Chile. Correspondencia a: Dra. Philippa Moore. E-mail: pmoore@med.puc.cl 
$20 \%$ de los casos se entregan las indicaciones sin discutir el plan de tratamiento ${ }^{12}$.

\section{¿NECESITAN MÁS INFORMACIÓN LOS PACIENTES?}

Los resultados de los primeros estudios, sobre los factores cognitivos involucrados en la relación médico-paciente, fueron inicialmente interpretados a favor de no gastar mucho tiempo en explicar o discutir el plan de manejo, asumiendo que los pacientes retenían poca información y que si se entregaban muchos detalles retenían aún menos ${ }^{13}$. Contradiciendo esta interpretación, Tuckett demostró que sólo 10\% de los pacientes falla en recordar todos los puntos claves que el médico le ha entregado y que cerca de 80\% manifestó encontrarse insatisfecho con respecto a la cantidad de información recibida y desearía recibir más detalles ${ }^{14}$

Algunos estudios muestran una asociación entre la entrega de información y una mayor satisfacción del paciente. La entrega de información se asociaría también a la mejora de algunos outcomes clínicos, tales como mejoría de síntomas, niveles de presión arterial, glicemia y requerimiento analgésico postoperatorio ${ }^{12,15,16}$. Esta evidencia realza la necesidad de incorporar esta variable a la práctica médica habitual, con el fin de optimizar los resultados buscados.

EL ROL DEL MÉDiCO COMO COMUNICADOR DE EVIDENCIA

La MBE se ha convertido, para muchos, en una excelente herramienta para la toma de decisiones y el transmitir la información obtenida al paciente sitúa al médico en el rol de comunicador de riesgo. El paso crucial en la entrega de información ocurre durante la interacción médico-paciente, donde un individuo (médico) interpreta $\mathrm{y}$ explica los datos clínicos a otro individuo (paciente). ¿Cuál es la mejor forma de entregar tal información?

En artículos anteriores hemos mostrado cómo evaluar la eficacia de una intervención y cómo aplicar estos resultados a un paciente determina$\mathrm{do}^{17,18}$. Imagine ahora que usted recibe a la señora R.M.C, de 50 años, hipertensa recién diagnosticada y quiere discutir con ella el manejo farmacológico de su hipertensión aplicando la evidencia existente.
¿De qué sirve que la mejor evidencia disponible sugiera que el medicamento A es mejor que el medicamento B para iniciar la terapia, si la paciente no entiende las consecuencias de su enfermedad, o prefiere tomar el medicamento B que se promociona en televisión? ¿Tiene importancia la manera en que le entregamos la información?

La tarea del médico no es meramente técnica, ya que, además de saber evaluar la literatura científica, debe explorar el significado de salud del paciente, incluyendo una evaluación de cuánta información quiere recibir éste y ofrecer un plan de manejo basado en ambos aspectos ${ }^{15}$. La noción de que las decisiones varían de circunstancia en circunstancia y de paciente en paciente recibe cada vez mayor atención. En este sentido, el estilo y método comunicacional cobran importancia porque pueden afectar el mensaje que el paciente recibe y, a su vez, influenciar las decisiones de éste ${ }^{19-21}$.

\section{EXPLICANDO RIESGOS}

En la práctica diaria los médicos se enfrentan frecuentemente con situaciones de incertidumbre, que requieren una evaluación de los beneficios y efectos adversos de las distintas opciones por parte del tratante y el paciente. Es así como se acuña el concepto de Comunicación de Riesgo, definido como un intercambio bidireccional de información y opinión, acerca del riesgo, que lleva a un mejor entendimiento y toma de decisiones en el manejo de una situación clínica ${ }^{22}$.

En este proceso nos encontramos con algunos obstáculos, como los problemas derivados del uso del lenguaje, ya sea al utilizar modalidades verbales o numéricas. Términos cualitativos como "infrecuente", "raro", "habitual", pueden tener un significado distinto, dependiendo del paciente o el contexto en el que se presenta el riesgo. Por ejemplo, la palabra "raro" suele ser interpretada como una probabilidad cercana al $24 \%$ por los pacientes y de $5 \%$ por los médicos, mientras que "frecuente" se interpreta de modo similar por $\operatorname{ambos}^{23,24}$. La interpretación de información numérica también es muy variable. Algunos autores han demostrado que ésta variará según como esté expresado un riesgo: la descripción de proporciones ("1 de cada 10 mujeres tendrá cáncer de mama") es considerado más orientado al paciente 
que las probabilidades (tener $10 \%$ de probabilidad de presentar cáncer de mama"), consideradas más difíciles de entender ${ }^{25}$. Sin embargo, el uso de proporciones tiende a producir el efecto de que el paciente atribuye el riesgo a otros, o sea, asume que está en el grupo sano ${ }^{26}$. Otros aspectos, como la edad, la cultura y el nivel educacional también pueden afectar la interpretación ${ }^{18,27}$.

La manera en la que se presenta la información sobre riesgo es otra variable a considerar. Un mismo dato se puede presentar de distintas formas, por ejemplo, al entregar cifras en función de mortalidad o sobrevida. Se ha observado que explicar los posibles daños facilita la aceptación de exámenes preventivos más que explicar los beneficios potenciales; y en pacientes que deben decidir por opciones de tratamiento más arriesgadas, resaltar las posibilidades de sobrevida es más persuasivo que mencionar las posibilidades de muerte ${ }^{20}$. Con el fin de evitar el sesgo derivado de la opinión del tratante, el cual puede no ser conciente, se sugiere presentar los datos de ambas maneras. También el orden en que se entrega la información y el material visual utilizado influye en la percepción del mensaje. Figuras humanas o caras serían más entendibles que gráficos y, si se usan gráficos, los gráficos de barras verticales son preferidos a los de barras horizontales ${ }^{27}$.

La transmisión de la información se complica más aún al incorporar algunos conceptos de MBE, como las medidas de efecto.

Términos como reducción de riesgo relativo (RRR), reducción de riesgo absoluto (RRA) y número necesario para tratar (NNT) han sido sindicados como los más importantes indicadores del significado clínico de una intervención terapéutica. Diversos estudios han demostrado que la información entregada en forma de riesgos relativos es más persuasiva que en forma de riesgos absolutos y que esto explicaría la preferencia de los pacientes por esta medida de efecto. Por otra parte, algunos autores han sugerido que presentar la información usando términos relativos es equívoco y exagera los resultados positivos, sobre todo si los beneficios absolutos son escasos o la intervención es a largo plazo ${ }^{18,28}$. Cifras absolutas o NNTs son difíciles de entender por los pacientes y suelen ser interpretados por éstos como "probabilidades remotas" ${ }^{18,29-32}$. Además, es difícil conciliar evidencia basada en estudios poblacionales con el cuidado de individuos que tienen necesidades, preocupaciones y expectativas únicas. Muchos de los outcomes que le importan al paciente, para la toma de decisiones, son subjetivos e intangibles (morir con dignidad, adecuada calidad de vida) y los pacientes valoran más la información individualizada que conocer riesgos poblacionales generales.

Una revisión sistemática sobre métodos de presentar evidencia clínica a los pacientes concluyó que falta evidencia sobre este tema. Basándose en literatura general sobre comunicación y en su propia experiencia clínica, los autores establecieron algunas recomendaciones sobre cómo comunicar la evidencia a los pacientes ${ }^{15}$ (Tabla 1). Otra estrategia para comunicar riesgo, descrita por Paling, es conocida como CARE 33 (Tabla 2).

\section{Tabla 1. Pasos para la discusión de evidencia médica con los pacientes 9}

- Comprender las vivencias y expectativas del paciente: Debiera realizarse una rápida, pero rutinaria, exploración de los miedos, ideas y expectativas del paciente (y su familia) acerca de una situación clínica, antes de discutir la evidencia médica, a fin de incorporar estas visiones a la toma de decisiones.

- Construir una alianza con el paciente: Incluir expresiones de empatía y entendimiento, verbalizando emociones, para fortalecer la confianza.

- Entregar la evidencia disponible, incluyendo una discusión balanceada sobre las incertidumbres: Hacer comparaciones con riesgos "de todos los días" o con situaciones familiares ayudan a la comprensión de la información. Anticipar dudas críticas.

- Entregar recomendaciones médicas: Después de integrar la evidencia con los valores del paciente.

- Evaluar la comprensión y grado de acuerdo de parte del paciente: Teniendo en cuenta la complejidad del tema tratado. 
Tabla 2. Estrategia CARE para comunicar riesgo ${ }^{22}$

- Citar el riesgo basal en términos generales: Favorezca el uso de proporciones más que probabilidades.

- Agregar a la explicación (en términos descriptivos) probabilidades estimadas de outcome negativos y positivos: Si está entregando información para favorecer una intervención preventiva, use términos en relación a los daños a evitar. Si está entregando información para favorecer un tratamiento, prefiera el uso de términos en función de sobrevida o ganancias.

- Reforzar efectividad con representaciones gráficas: Uso de figuras humanas y, si usa gráficos, idealmente de barra.

- Expresar aliento y esperanza.

No existe un abordaje único que sirva para todos los pacientes. Por esto, los médicos requieren de una variedad de técnicas para comunicar la evidencia acerca de diagnóstico, tratamiento, riesgo y pronóstico, así como distintas maneras de verificar que la información ha sido comprendida. Lamentablemente, hay pocos estudios que aportan evidencia de buena calidad acerca de la manera más apropiada en que los médicos pueden incorporar la transmisión de evidencia clínica en la práctica diaria y no existe consenso acerca de los métodos más apropiados para entregar la información 15,34,35.

Una consecuencia interesante de intercambiar información con los pacientes es la de entregarles el poder de tomar sus propias decisiones o, al menos, de participar activamente en el proceso, lo que conlleva compartir las consecuencias. Si los pacientes esperan y se espera de ellos que tomen esas decisiones, la carga de la responsabilidad por aceptar la incertidumbre también será compartida. El aumento de la participación de los pacientes en la toma de decisiones en el cuidado de su salud, modalidad de relación médico-paciente llamada toma de decisiones compartidas, está asociado con mayor confianza en la decisión tomada, mayor satisfacción con la atención recibida, mayor adherencia a los planes médicos, disminución de los costos de tratamiento y finalmente mejor calidad de vida y menor mortalidad ${ }^{15,16,36-38}$.

\section{EN RESUMEN}

La comunicación de los riesgos de enfermedades y tratamientos es central en la relación médicopaciente y es un determinante esencial de la satisfacción de este último.

Contamos con información limitada acerca de la forma más adecuada de presentar y discutir los riesgos y beneficios del cuidado de salud de un individuo.

Futuras investigaciones ayudarán a definir los mecanismos óptimos para incorporar la transmisión de evidencia a la práctica clínica. Actualmente, la combinación de técnicas cuantitativas y cualitativas, sumada a la presentación gráfica de los datos, pareciera ser lo más útil para explicar riesgos a los pacientes.

Cómo y cuánta evidencia entregar a los pacientes y sus familias dependerá del tipo de información, del médico, del paciente y su entorno. La discusión de la evidencia será más necesaria cuando el tema es importante para el paciente, cuando la evidencia es ambigua o los outcomes inciertos o cuando la evidencia es clara pero el paciente o su familia difieren sustancialmente del médico en su interpretación.

El desafío entonces, consiste en transmitir el mensaje al paciente de manera que, si éste lo desea, pueda participar en forma más activa en la toma de decisiones. 


\section{REFERENCIAS}

1. Letelier SL, Moore P. Introducción. Rev Méd Chile 2003; 131: 947-8.

2. Letelier SL, Moore P. La medicina basada en evidencia: Visión después de una década. Rev Méd Chile 2003; 131: 939-46.

3. Engel GL. From biomedical to biopsychosocial. Being scientific in the human domain. Psychosomatic 1997; 38: 521-8.

4. Sweeney KG, Macauley D, Pereira Gray D. Personal significance: the third dimension. The Lancet 1998; 351: 134-6.

5. WaitZkin H. Doctor-patient-communication: clinical implications of social scientific research. JAMA 1984; 252: 2441-6.

6. Svarstad BL. The doctor-patient encounter: an observational study of communication and outcome doctoral dissertation, University of Wisconsin, Madison 1974.

7. Deber R, Kraetschmer N, Irvine J. What role do patients wish to play in treatment decision making? Arch Intern Med 1996; 156: 1414-20.

8. Strull W, Lo B, Charles G. Do patients want to participate in medical decision making? JAMA 1984; 252: 2990-4.

9. Braddock CH, Finn SF, Levinson W, Jonsen AR, Pearlman RA. How doctors and patients discuss routine clinical decisions: informed decision making in the outpatient setting. J Gen Intern Med 1997; 12: 339-45.

10. Beckmann HB, Frankell RM. The effect of physician behavior on the collection of data. Ann Intern Med 1984; 101: 692-6.

11. Korsch B, Putnam S, Frankel F, Roter D. An overview of research on medical interviewing. In: Lipkin M Jr, Putnam SM, Lazare A, editors. The medical interview. Clinical care, education and research. New York: Springer-Verlag, 1995.

12. Silverman J, Kurtz S, Draper J. Skills for communicating with patients. 1998 Radcliffe Medical Press, Oxon.

13. Ley P. Doctor-patient communication: some quantitative estimates of the role of cognitive factors in non-compliance. J Hypertens Suppl 1985; 3: S51-5.

14. Tuckett D, Williams A. Approaches to the measurement of explanation and information-giving in medical consultations: a review of empirical studies. Soc Sci Med 1984; 18: 571-80.
15. Ebstein RM, Alper BS, Quill TE. Communicating evidence for participatory decision making. JAMA 2004; 291: 2359-66.

16. Frosch DL, Kaplan RM. Shared decision making in clinical medicine: past research and future directions. Am J Prev Med 1999; 17: 285-94.

17. Rivera S, Larrondo F, Ortega JP. Evaluación de los resultados en un artículo sobre tratamiento. Rev Méd Chile 2005; 133: 593-6.

18. Rada G, Andrade A. ¿Debo aplicar los resultados de este estudio a mi paciente? Rev Méd Chile 2006; 134: 113-7.

19. Ghosн AK, Ghosн K. Translating evidence-based information into effective risk communication: Current challenges and opportunities. J Lab Clin Med 2005; 145: 171-80.

20. Edwards A, Elwyn G, Covey J, Mathews E, Pill R. Presenting risk information - a review of the effects of framing and other manipulations on patient outcomes. J Health Comm 2001; 6: 6182.

21. Sarfati D, Howden-Chapman P, Woodward A, SAlMOND C. Does the frame affect the picture? A study into how attitudes to screening for cancer are affected by the way benefits are expressed. J Med Screening 1998; 5:137-140.

22. Edwards A, ElWyn G, Mulley A. Explaining risks: turning numerical data into meaningful pictures. BMJ 2002; 324: 827-30.

23. Sutherland HJ, Lockwood GA, Tritchler DL, Sem F, Brooks L, Till JE. Communicating probabilistic information to cancer patients: is there "noise" on the line? Soc Sci Med 1991; 32: 725-31.

24. Cohn LD, Schydlower M, Foley J, Copeland RL. Adolescents misinterpretation of health risk probability expressions. Pediatrics 1995; 95: 713-6.

25. YAmagishi K. When a $12.86 \%$ mortality is more dangerous than 24.14\%: implications for risk communication. Appl Cogn Psychol 1997; 11: 495506.

26. Shapira mM, Nattinger AB, Mchorney CA. Frequency or probability? A qualitative study of risk communication formats used in health care. Med Decis Making 2001; 21: 459-67.

27. Mazur DJ, Merz JF. How the manner of presentation of data influences older patients in determining their treatment preferences? J Am Geriatr Soc 1993; 41: 223-8 
28. Chao C, Studts JL, Abell T, Hatley T, Roetzer L, Dinne $S$ ET AL. Adjuvant chemotherapy for breast cancer: how presentation of recurrence risk influences decision making? J Clin Oncol 2003; 21: 4299-305.

29. Sheridan SL, Pignone MP, Lewis CL. A randomized comparison of patients' understanding of number needed to treat and other common risk reeducation formats. J Gen Intern Med 2003; 18: 884-92.

30. Hux Je, Naylor CD. Communicating the benefits of chronic preventive therapy: Does the format of efficacy data determine patient's acceptance of treatment? Med Decis Making 1995; 15: 152-7.

31. Kristiansen IS, Gyrd-Hansen D, Nexoe J, Nielsen JB. Number needed to treat: easily understood and intuitively meaningful? Theoretical considerations and a randomized trial. J Clin Epidemiol 2002; 55: 888-92.

32. Pearson SD, Golrnan L, Garcia TB, Coo EF, Lee TH. Physician response to a prediction rule for the triage of emergency department patients with chest pain. J Gen Intern Med 1994; 9: 241-7.

33. PALING J. Strategies to help patients understand risks. BMJ 2003; 327: 745-8.
34. Edwards A, Unigwe S, Elwyn G, Hood K. Personalized risk communication for informed decision making about entering screening programs. (Review). The Cochrane Database of Systematic Reviews 2003, Issue1.

35. Ghosh AK, ERwin P, GHosh K. Effective risk communications: multiple modalities, nuclear consensus. A review of literature. J Invest Med 2002; 50: 182.

36. Lang F, Floyd MR, Beine KL, Buck P. Sequenced questioning to elicit the patients perspective on illness: effects on information disclosure, patients satisfaction and time expenditure. Fam Med 2002; 34: 325-30.

37 Slowther A, Ford S, Schofield T. Ethics of evidence based medicine in the primary care setting. Symposium on Evidence Based Medicine. J Med Ethics 2004; 30: 151-5.

38. Betz Brown J, Boles Myde, Mullooly JP, Levinson W. Effect of clinician communication skills training on patient satisfaction. A randomized, controlled trial. Ann Intern Med 1999; 131: 822-9. 